\title{
Reescritura en pareja: una tarea de aprendizaje inicial de lo escrito
}

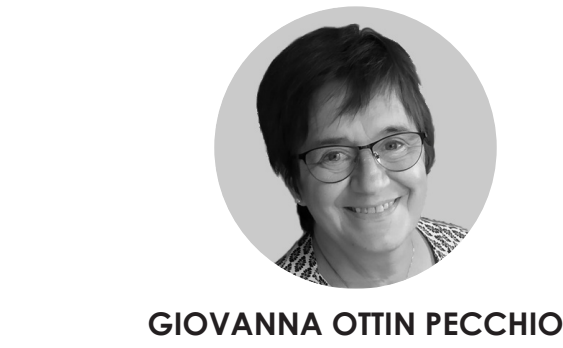

LANGUAGE \& EDUCATION RESEARCH GROUP

giottin@gmail.com

$\mathrm{E}$ n este artículo se presenta una tarea de reescritura de textos en pareja de dos niños en los momentos iniciales del proceso de aprendizaje del lenguaje escrito (entre 5 y 7 años). Los objetivos de este artículo son los siguientes: (i) mostrar la importancia de las decisiones pedagógicas tomadas por el docente sobre un tipo de tarea que permite observar los procesos de aprendizaje que permanecerían ocultos en otras situaciones; y (ii) llamar la atención sobre las capacidades que los aprendices pueden llegar a mostrar tanto en las interacciones verbales entre ellos como en la producción de textos reescritos compartidos, cuando se les da la oportunidad de hacerlo. Por último, se resumen los beneficios que la utilización de este tipo de tareas puede tener en la alfabetización inicial.

\section{PALABRAS CLAVE:}

Escritura; tarea interactiva; lectura en voz alta; lenguaje escrito; alfabetización inicial.

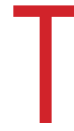

his paper presents a re-writing task to be carried out in pairs by 5 to 7 -year old early readers and writers. The paper aims to (i) show the importance of the pedagogical decisions undertaken by a teacher who choose to implement tasks which allow her to observe learning processes that remain hidden under other circumstances; and (ii) underscore the advanced capacities that the learners may show in their conversations and in their shared product texts when they are given the opportunity to do so. The paper concludes with a set of implications for classroom practice.

\section{KEYWORDS:}

Rewriting; interaction task; reading out loud; written language; initial literacy. 


\section{Introduction}

E n este artículo se presenta una descripción de una tarea de reescritura de textos en pareja de dos niños en los momentos iniciales de su proceso de aprendizaje del lenguaje escrito (entre 5 y 7 años). Los objetivos de esta descripción son

(i) mostrar la importancia de la decisión pedagógica sobre el tipo de tarea con la finalidad de observar procesos de aprendizaje frecuentemente ocultos en otras situaciones, y al mismo tiempo

(ii) evidenciar las capacidades infantiles tanto en las interacciones verbales como en la producción de textos en situaciones de hacer una imitación en diferido en la actividad de reescribir un texto conocido y compartido.

El ciclo de la tarea comprende tres actividades:

- lectura en voz alta del cuento por parte del adulto,

- discusión en pareja de aprendices sobre el contenido (el qué escribir) y la forma (el cómo escribir), y finalmente,

- reescritura del cuento por una pareja de dos niños.

En la exposición que sigue se mostrará: 1) la función de la lectura en voz alta y 2) las razones que hacen de la reescritura un instrumento que activa importantes mecanismos de aprendizaje inicial del lenguaje. Se considerará luego 3) el valor del trabajo interactivo en la construcción conjunta de un texto narrativo a partir de un texto-modelo (o textofuente) de literatura infantil y se presentará 4) la descripción detallada de la tarea, para finalmente concluir resumiendo las principales ventajas de la utilización en el aula de este tipo de tareas.

\section{La lectura en voz alta}

$\mathrm{P}$ ara la población no escolarizada, y para los niños al principio de la alfabetización, la lectura en voz alta (LVA) ha sido durante siglos y es el mejor procedimiento de difusión del lenguaje escrito (Frenk, 2004; Teberosky y Jarque, 2014), ya que ha permitido y permite la oralización de lo escrito o, como bien se ha dicho, permite "que lo letrado se pueda transmitir oralmente" (Teberosky, 2011).

La relación entre alfabetización emergente y lectura en voz alta a un aprendiz todavía no completamente alfabetizado, ha sido ampliamente documentada, $\mathrm{y}$ ha sido definida como "la quintaesencia de los eventos letrados" (Pellegrini, 1996). Investigaciones en este campo han mostrado sus beneficios en aspectos lingüísticos y cognitivos del desarrollo infantil (Teberosky y Jarque, 2015). En particular, se ha descrito que la actividad dialógica de leer en voz alta, es decir, la actividad oral que se desarrolla entre dos (o más) personas sobre la lectura y la visualización de un texto, favorece:
- el desarrollo del conocimiento sobre lo impreso (función de los libros, identificación de las letras, dirección de la escritura etc.),

- la ampliación del vocabulario (Farrant y Zubrick, 2013),

- la familiarización con el lenguaje letrado y la incorporación de estructuras sintácticas nuevas (Bus, van Ijzendoorn y Pellegrini, 1995),

- la comprensión lectora y el reconocimiento del registro propio de lo escrito, ya que a través de ella los niños aprenden a evitar construcciones que sólo se dan en el habla espontánea.

La propuesta didáctica que se plantea aquí subraya también la importancia que tiene la elección de los libros que se leen a los niños y se proponen para el trabajo escolar. Dicha elección está guiada por dos objetivos principales: por un lado, responde a la exigencia de tener en cuenta la realidad psicológica del niño en función de sus conocimientos y de sus capacidades como aprendiz lector y escritor; por el otro propone que los textos se elijan según los aspectos lingüísticos o estructurales que se quieren trabajar y poner en evidencia.

La preferencia va para libros de calidad literaria: libros tradicionales de literatura infantil y libros contemporáneos, en particular libros tipo álbum ${ }^{1}$, es decir, textos reales, y no manuales de lectura que están fuera de todo contexto más allá del ámbito escolar ("textos huérfanos", según Bruner). A partir de la lectura en voz alta del texto puede realizarse la reescritura, como mímesis del texto original.

\section{La reescritura}

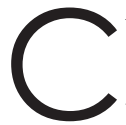

uando hablamos de reescritura nos referimos a una actividad de imitación diferida o reproducción de un texto precedentemente leído y escuchado, donde el texto reescrito (o texto resultante) puede mantener diferentes grados de equivalencia con el texto original (o texto fuente).

La reescritura de un texto previamente leído a los niños es una práctica poco habitual en el aula del último año de Infantil (5 años) y en la del primer grado de Primaria, debido principalmente a que se considera que el niño debe dominar la escritura alfabética de las palabras y de las frases antes de enfrentar la escritura de un texto. En la actualidad hay evidencias empíricas que cuestionan esta posición: los niños pueden reproducir y escribir textos si están en contacto con textos, lectores y escritores (Sepúlveda y Teberosky, 2009; Teberosky y Sepúlveda, 2008). Se ha observado que, bajo estas condiciones, ellos logran reproducir la forma propia de la organización del "lenguaje que se escribe" (o "lenguaje dominguero", según la denominación propuesta por BlancheBenveniste, 1982), es decir, logran conservar los registros y las modalidades del discurso escrito. En particular, si el discurso se desarrolla alrededor de cuentos de narrativa infantil, los niños son capaces de utilizar los tiempos verbales típicos de la narración, como el imperfecto y el pasado indefinido, sujetos 
invertidos en marco de cita, frases adverbiales, expresiones o palabras literarias, citaciones, fórmulas para el inicio o el final (Sepúlveda, 2012).

Al mismo tiempo la actividad de reescritura favorece operaciones lingüísticas más o menos miméticas respecto al texto fuente, como la citación literal, la paráfrasis o la reformulación. Dichas operaciones permiten ir más allá de la interpretación del texto: por ejemplo, conservar las "mismas palabras" del texto modelo, es decir la literalidad del texto, su citación, es un logro que depende de la alfabetización y de la comprensión de la naturaleza permanente de la escritura (Lee, Torrance y Olson, 2001). En este sentido, la recuperación del texto requiere un gran esfuerzo de memoria y de atención por parte de los aprendices.

Por otro lado, según varios autores, también la reformulación y la paráfrasis solicitan el funcionamiento de la lengua y favorecen su adquisición: sintetizar, ampliar, corregir o autocorregirse, son acciones que permiten que el niño se apropie de lo que oye, transformándolo y usándolo (Martinot, 2000).

Finalmente la introducción de la reescritura en la enseñanza/ aprendizaje inicial demuestra ser un instrumento que facilita la construcción y redacción del texto, ya que evita que los niños tengan que enfrentarse al esfuerzo adicional de construir una historia ex novo: si el contenido y la estructura de la historia ya están dados, el escritor debutante tiene la posibilidad de concentrase más en la elección del léxico, de la ortografía o de la sintaxis, es decir de atender a la forma del texto (Ferreiro, Pontecorvo, Ribeiro Moreira, García Hidalgo, 1996).

\section{La reescritura colaborativa entre pares}

A ntes de la reescritura de un texto, la lectura en voz alta suele dar lugar a discusiones y comentarios sobre lo leído y escuchado. En la mayoría de los casos, los intercambios que suceden son conducidos por el maestro. De hecho la propuesta de intervención en aula a la cual nos referimos prevé un trabajo sistemático y guiado por el adulto: lo que se intenta es controlar las condiciones de recepción de los aprendices lectores y escritores para llevarlos a comprender y apropiarse del texto como discurso y de sus características (véase el trabajo de Ana Teberosky y de su equipo en https://www.aprendretextos.com/ y http:// espacodeleitura.labedu.org.br/).

Al contrario, la reescritura colaborativa entre pares, a diferencia de la situación controlada por el maestro, tiene relativamente pocos precedentes de estudios empíricos y muy poca presencia en la práctica escolar. Esto se debe a que, por un lado, un punto de vista bastante difundido en las aulas es considerar la redacción de un texto como una actividad principalmente solitaria; por otro lado, porque los maestros, sobre todo en contextos de enseñanza tradicional, refieren una cierta aprensión en aceptar un tipo de trabajo que temen no poder controlar.
Sin embargo, se ha mostrado que la construcción del significado y la estructuración de la narración son observables a través del trabajo en parejas formadas por iguales. La colaboración parece tener un impacto significativo sobre el mantenimiento de la coherencia, de la cohesión y de la referencia (Preece, 1992), la apropiación del lenguaje descontextualizado, es decir el lenguaje no vinculado al contexto y a la experiencia inmediata, (Jones, 2003; Hall, 2000), del registro propio del lenguaje escrito (Sepúlveda y Teberosky 2011) y sobre el aprendizaje de habilidades narrativas (Küntay y Senay, 2003).

Además, el trabajo en pareja parece ser un potencial dinamizador de los conocimientos y de las reflexiones sobre la lengua (Escobar Urmeneta 2004; 2014), ya que es a través del "dialogo colaborativo" que los alumnos pueden ir más allá de sus propias competencias individuales (Swain 2000).

En esta misma línea se ha mostrado también que los niños pueden colaborar para seleccionar lo que tienen que escribir, alternarse en el dictado y en la redacción, así como representarse las intenciones del compañero para evaluarlas (Alam y Rosemberg, 2015; Wang y Cassel, 2003). El hablar para ponerse de acuerdo en la redacción de un texto, comentarlo, re-leerlo, evaluarlo, es decir hablar sobre el texto, su forma, su contenido y su desarrollo permite a los niños compartir procedimientos y favorece la actividad metalingüística y metacognitiva. Las observaciones conducidas muestran un gran repertorio de actividades lingüísticas que en situaciones tradicionales son conducidas por los maestros: repeticiones de las unidades dictadas, deletreo de palabras, sugerencias técnicas o de contenido, propuestas de copia de modelos gráficos, entre otros. También son observables dos posiciones enunciativas que se alternan: una más cercana a la de escritor, es decir a la de quien está implicado en la elaboración del texto, la otra más cercana a la del lector, que juzga el resultado.

A través de esta actividad se intenta llevar al niño a tratar el texto no sólo semánticamente, sino también literalmente para acercarse a la forma y al registro de la lengua escrita. A través de la reescritura colaborativa los niños pueden aprender a:

- diferenciar entre el hablar para dictar y el hablar simplemente, es decir entre hablar en una conversación y hablar para escribir: la elaboración del discurso es más monológica y no todo lo que se dice hay que escribirlo;

- distinguir entre lo que ya se ha escrito y lo que falta por escribir;

- volver sobre lo que ya se ha dicho: reformular o redecir para dictar lo mismo y entender la estabilidad del escrito;

- segmentar, al dictar, en unidades diferentes, dependiendo de los tiempos de escritura del compañero y de las pausas;

- hacer corresponder lo que se dice con lo que se escribe, a través de la observación del acto de escritura del compañero; 
- construir competencias metalingüísticas: usar palabras para hablar sobre las palabras ("nombre", "cuento", " texto", "título" etc.)

- memorizar el texto fuente y sus secuencias;

- recuperar lo que se ha dictado a través de la lectura y re-lectura.

\section{La tarea paso a paso: descripción y aplicación en el aula}

Formación de las parejas de niños.

\section{Primer paso: la lectura en voz alta}

Lectura enfática por parte del adulto, respectando pausas y prosodia. En particular en los libros tipo álbum la disposición gráfica de las líneas ayuda a evidenciar el sentido de lo que se lee. En el ejemplo que sigue se muestra este tipo de disposición (llamada también línea de sentido, o disposición por cola et commata):

\section{Había una vez un príncipe \\ que quería casarse con una princesa \\ pero quería sobre todo \\ que fuese una princesa de verdad}

(Adaptación del cuento La Princesa y el guisante de H.C. Andersen)
La lectura se propone a través de la visualización en pantalla grande para que pueda ser mostrado el texto (su disposición gráfica, su tipografía y formato) conjuntamente con las ilustraciones. Una opción alternativa, consiste en poner a disposición de los niños el libro para que puedan hojearlo.

\section{Segundo paso: la consigna para la tarea}

a. Se pide a los niños que reescriban el cuento recién leído y se les dice que redacten el texto lo mejor que puedan, sin inventar nada nuevo y tratando de recordar las palabras del texto.

b. Se invita a los niños a colaborar entre ellos y alternarse en la escritura y en el dictado: cada uno puede dar su opinión, hacer una propuesta o corregir el otro en cualquier momento de la tarea. Si se desea estudiar el proceso de reescritura compartida es necesario grabar las conversaciones que se desarrollan.

c. A cada pareja se le proporcionan hojas blancas formato A4 y dos lápices de colores diferentes: cada niño escribirá su parte con un color diferente del compañero, de manera que las contribuciones de cada uno se distingan con claridad. Se les pide que si se equivocan no borren con goma lo escrito, sino que lo tachen. De esta manera el maestro puede observar el proceso seguido por el niño para llegar a la versión final.
Figura 1. Texto reescrito en pareja por dos aprendices (Fuente: Ottin Pecchio 2018).

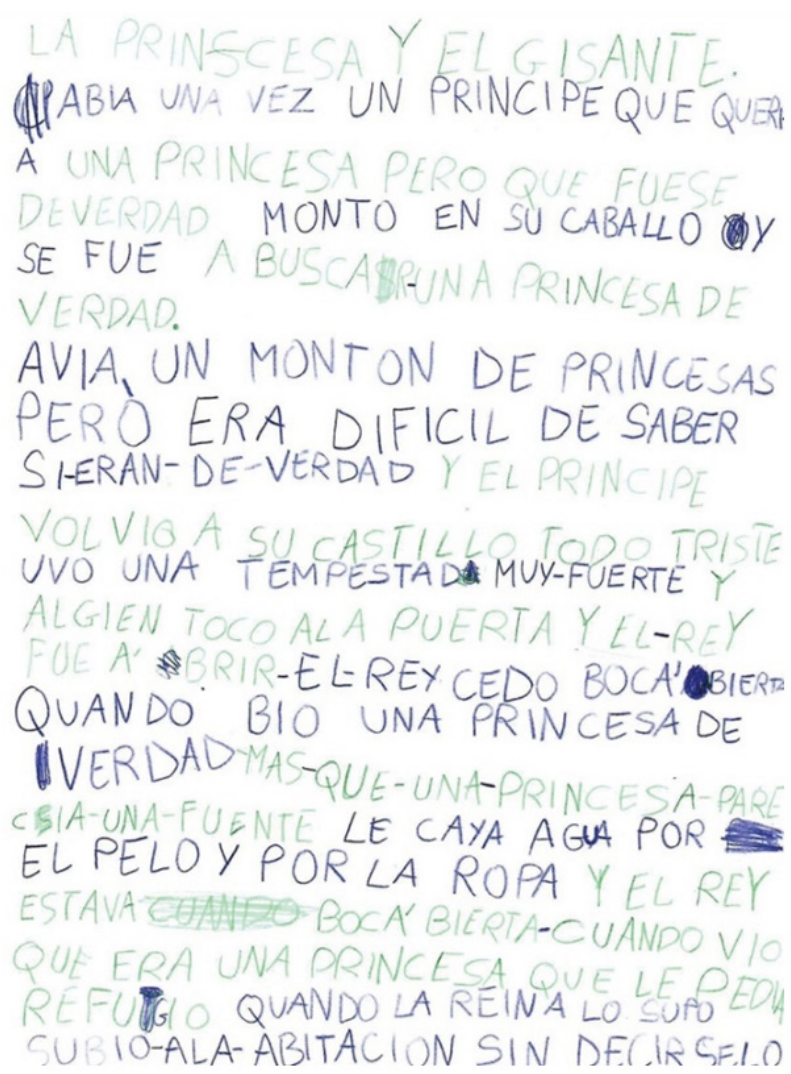




\section{Implicaciones didácticas}

$\mathrm{O}$ ttin Pecchio (2018) aborda en su estudio empírico este tipo de tareas. De dicho estudio procede la Figura 1 que muestra el resultado final del texto reescrito por dos aprendices de 6 años sobre el cuento de la Princesa y el guisante.

La observación atenta de este y otros textos similares, así como la escucha de las conversaciones de los niños mientras escriben ayudan a reflexionar sobre los beneficios de la utilización en el aula de este tipo de tareas. Así, del estudio se concluyen las siguientes implicaciones didácticas para la enseñanza inicial de lo escrito. Entre estas:

- La importancia de considerar la "agenda" de los niños, es decir sus exigencias de aprendizaje. A través de las discusiones sobre cómo escribir el texto los niños pueden poneren evidencia qué temáticas priorizan, (por ejemplo, la puntuación, la ortografía, la segmentación del texto, el léxico, la organización del discurso etc.): conocer estas temáticas otorga la posibilidad al maestro de organizar la enseñanza en torno a lo que resulta más problemático para los aprendices.

- La variedad de acciones en torno al texto. Una aproximación de este tipo favorece una gran cantidad de actividades entre oralidad y escritura: argumentar, citar, comentar, comparar, denominar, dictar, escribir, explicar, evaluar, formular, leer, reescribir, reformular, re-leer, reparar, repetir, entre otras.

- La consideración de que esta tarea, con la alternancia de autores y colores en la escritura, puede ayudar los niños a identificar unidades lingüísticas. En el citado estudio de Ottin Pecchio (2018) se demuestra que el cambio de mano en la redacción del texto compartido no es aleatorio, sino que evidencia diferentes unidades lingüísticas y textuales: palabras, líneas, oraciones, párrafos entre otras. La posibilidad de que dichas unidades queden materialmente registradas, a través de la alternancia de colores en el texto, las convierte en observables, no sólo para el maestro, sino también para los aprendices. Este proceso favorece en ellos un cierto nivel de conceptualización sobre la segmentación de lo escrito en su relación con lo oral.

- La necesidad de cambiar los instrumentos enseñanza o materiales educativos, y la utilización que se hace de ellos. Así, los resultados obtenidos avalan la utilización de libros con calidad literaria sobre los "textos huérfanos" propuestos por los manuales, por un lado, y de actividades en torno al texto, que transcienden la mera ejercitación, tales como lectura en voz alta, la interacción entre pares o la escritura compartida. La combinación de este tipo de materiales y actividades de alto reto cognitivo e interacción real generan entornos potencialmente adquisitivos de elementos lingüísticos y discursivos.

\section{${ }^{66}$ Una tarea interactiva de reescritura estimula a los niños a "hablar sobre" la lengua, sus normas y convenciones y en cuanto tal favorece el desarrollo de la conciencia metalingüística y del metalenguaje.}

\section{Conclusión}

Una tarea interactiva de reescritura estimula a los niños a "hablar sobre" la lengua, sus normas y convenciones y en cuanto tal favorece el desarrollo de la conciencia metalingüística y del metalenguaje. Por ello resulta esencial proponer tareas de aprendizaje (y no sólo de ejercitación) que planteen un problema a resolver. La reescritura compartida entre dos niños ha demostrado reunir estas características.

\section{Notas}

1 El libro tipo álbum narrativo tiene una distribución equitativa entre texto e ilustración, que permite combinar las formas verbal y visual cooperando a la creación de sentido (Colomer, 2002). Se caracteriza por riqueza lingüística y variedad tipográfica que, junto con las disposiciones en la página, ayudan a poner en relieve el texto y sus partes.

\section{Bibliografia}

Alam, F., \& Rosemberg, C. R. (2015). Un análisis del proceso de construcción interaccional en narrativas de ficción entre pares. Papeles de Trabajo sobre Cultura, Educación y Desarrollo Humano, 11(1), 1-16. Recuperado de http:// hdl.handle.net/11336/32923

https://doi.org/10.16888/interd.2015.32.1.2

Blanche-Benveniste, C. (1982). La escritura del lenguaje dominguero. En Ferreiro, E. \& Gómez, M. (Eds.), Nuevas perspectivas sobre los procesos de lectura y escritura (pp.247-270). México: Siglo XXI. 
https://doi.org/10.35537/10915/74969

Bus, A. G., Van Ijzendoorn, M. H., \& Pellegrini, A. D. (1995). Joint book reading makes for success in learning to read: A meta-analysis on intergenerational transmission of literacy. Review of educational research, 65(1), 1-21.

https://doi.org/10.3102/00346543065001001

Colomer, T. (2002) (Ed.). Siete llaves para valorar las historias infantiles. Madrid: Fundación G. Sánchez Reuipérez.

https://doi.org/10.5944/epos.19.2003.10376

Escobar Urmeneta, C. (2004). Interacción oral y aprendizaje de lenguas extranjeras. Mosaico, 8, 23-30.

Escobar Urmeneta, C. (2014). Conversar más y mejor en la clase de inglés. Para aprender mejor y llegar más lejos. Aula de innovación educativa 237, 12-17.

Farrant, B. M., \& Zubrick, S. R. (2013). Parent-child book reading across early childhood and child vocabulary in the early school years: Findings from the Longitudinal Study of Australian Children. First Language, 33(3), 280-293.

https://doi.org/10.1177/0142723713487617

Ferreiro, E., Pontecorvo C., Ribeiro Moreira, N. \& García Hidalgo, I. (1996). Caperucita Roja aprende a escribir. Barcelona, Gedisa.

https://doi.org/10.24201/nrfh.v46i2.2066

Frenk, M. (2004). Oralidad y escritura y lectura. En Rico F. (Ed.). Don Quijote de la Mancha (pp. 1138-1144). Madrid y México: Real Academia Española, Asociación de Academias de la Lengua Española, Editorial Alfaguara.

https://doi.org/10.5944/epos.25.2009.10627

Hall, N. (2000). Literacy, play, and authentic experience. En Roskos, K. \& Christie, J. (Eds.). Play and literacy in early childhood (pp. 189-204). Mahwah, NJ: Erlbaum.

Jones, I. (2003). Collaborative writing and children's use of literate language: A sequential analysis of social interaction. Journal of Early Childhood Literacy, 3(2), 165-178.

https://doi.org/10.1177/14687984030032003

Küntay, A.C. \& Senay, I. (2003). Narratives beget narratives: Rounds of stories in Turkish preschool conversations. Journal of Pragmatics, 35, 559-587.

https://doi.org/10.1016/s0378-2166(02)00129-7

Lee, E. A., Torrance, N., Olson D.R. (2001) Young children and the say/mean distinction: verbatim and paraphrase recognition in narrative and nursery rhyme contexts. Journal of Child Language, 28, 531-543.

https://doi.org/10.1017/s0305000901004755

Martinot, C. (2000). Étude comparative des processus de reformulations chez des enfants de 5 à 11 ans. Langages, 34 (140), 92-123.

https://doi.org/10.3406/lgge.2000.2392

Ottin Pecchio, G. (2018). Oralidad y escritura compartida en la alfabetización inicial. Estudio en un contexto multilingüe. Tesis doctoral. Universitat Autònoma de Barcelona.

Pellegrini, A.D. (1996). Observing Children in the Natural Worlds: A Methodological Primer. Mahwah, NJ: Erlbaum.

Preece, A. (1992). Collaborators and Critics: the nature and effects of peer interaction on children's conversational narratives. Journal of Narrative and Life History, 2(3), 277-292.

https://doi.org/10.1075/jnlh.2.3.06col

Sepúlveda, A. (2012). El aprendizaje inicial de la escritura de textos como (re)escritura. Tesis doctoral. Universidad de Barcelona.

https://doi.org/10.14483/udistrital.jour.enunc.2014.1.a02

Sepúlveda, A. \& Teberosky, A. (2011). El lenguaje en primer plano en la literatura infantil para la enseñanza y el aprendizaje inicial del lenguaje escrito. Cultura $y$ Educación, 26(1), 23-42.

https://doi.org/10.1174/113564011794728560

Swain, M. (2000). The output hypothesis and beyond: Mediating acquisition through collaborative dialogue. Sociocultural theory and second language learning, 97114.

Teberosky, A. (2011). Desde la literatura al aprendizaje del lenguaje y su escritura. Actas del Simposio Internacional. La literatura que acoge: infancia, inmigración $y$ lectura. Organizado por el Grupo de Investigación de Literatura Infantil y Juvenil y Educación Literaria del Departamento de Didáctica de la Lengua, de la Literatura y de las Ciencias Sociales de la Universidad Autónoma de Barcelona. Recuperado de www.aprendretextos.com https://doi.org/10.15645/alabe.2012.5.2

Teberosky, A. \& Jarque, M. J. (2014). Interacción y continuidad entre la adquisición del lenguaje y el aprendizaje de la lectura y la escritura. Ruta maestra, 8 , 21-26. 
Teberosky, A. \& Jarque, M. J. (2015). Aprender lenguaje entre 0 y 5 años. En http://aprenderlinguagem.org.br/

Teberosky, A. \& Sepúlveda, A. (2008). La literatura en la alfabetización inicial. En Actas V Congreso Internacional de Psicología y Educación: Los retos del futuro (pp. 990996). Oviedo: Universidad de Oviedo.

https://doi.org/10.1174/113564011794728560

Teberosky, A. \& Sepúlveda, A. (2009). El texto en la alfabetización inicial. Infancia y Aprendizaje, 32(2), 199218.

https://doi.org/10.1174/021037009788001770

Wang A. \& Cassel J. (2003). Co-authoring, Corroborating, Criticizing: Collaborative Storytelling between Virtual and Real Children. Paper presented at Workshop of Educational Agents: More than Virtual Tutor. Vienna. Recuperado de https://pdfs.semanticscholar.org/42a1/ b8831174be0850cdd00314eb4971fe88ef70.pdf 